\title{
Structural Aspects of Photopharmacology: Insight into the Binding of Photoswitchable and Photocaged Inhibitors to the Glutamate Transporter Homologue
}

Valentina Arkhipova, Haigen Fu, Mark W. H. Hoorens, Gianluca Trinco, Lucien N. Lameijer, Egor Marin, Ben L. Feringa, Gerrit J. Poelarends, Wiktor Szymanski,* Dirk J. Slotboom,* and Albert Guskov*

Cite This: J. Am. Chem. Soc. 2021, 143, 1513-1520

Read Online

ACCESS

Llll Metrics \& More

Article Recommendations

Supporting Information

ABSTRACT: Photopharmacology addresses the challenge of drug selectivity and side effects through creation of photoresponsive molecules activated with light with high spatiotemporal precision. This is achieved through incorporation of molecular photoswitches and photocages into the pharmacophore. However, the structural basis for the light-induced modulation of inhibitory potency in general is still missing, which poses a major design challenge for this emerging field of research. Here we solved crystal structures of the glutamate transporter homologue $\mathrm{Glt}_{\mathrm{Tk}}$ in complex with photoresponsive transport inhibitors-azobenzene derivative of

\section{X-ray structural basis for photopharmacology}

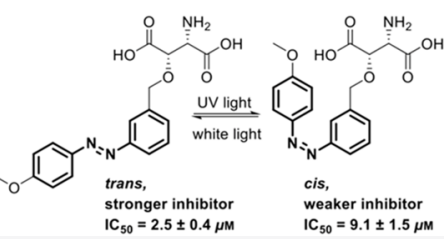

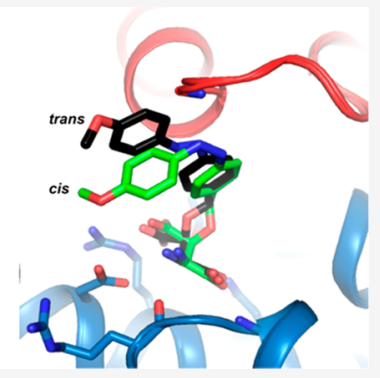
TBOA (both in trans and cis configuration) and with the photocaged compound ONB-hydroxyaspartate. The essential role of glutamate transporters in the functioning of the central nervous system renders them potential therapeutic targets in the treatment of neurodegenerative diseases. The obtained structures provide a clear structural insight into the origins of photocontrol in photopharmacology and lay the foundation for application of photocontrolled ligands to study the transporter dynamics by using time-resolved X-ray crystallography.

\section{INTRODUCTION}

Photopharmacology is an emerging field that aims to control the action of bioactive molecules by light, enabling spatiotemporal precision of pharmacological treatment, which can reduce undesired interactions and side effects and prevent the development of resistance. ${ }^{1-6}$ Because the majority of drug targets are membrane proteins, ${ }^{7}$ there is an increasing interest in the application of photoregulated compounds in membrane protein research, with recent examples including GPCRs, ${ }^{8-10}$ ion channels, ${ }^{11}$ receptors, ${ }^{12}$ and receptor-linked enzymes. ${ }^{13}$

Photopharmacology relies on the introduction of molecular photoswitches, such as azobenzene ${ }^{14}$ (Figure 1, highlighted in

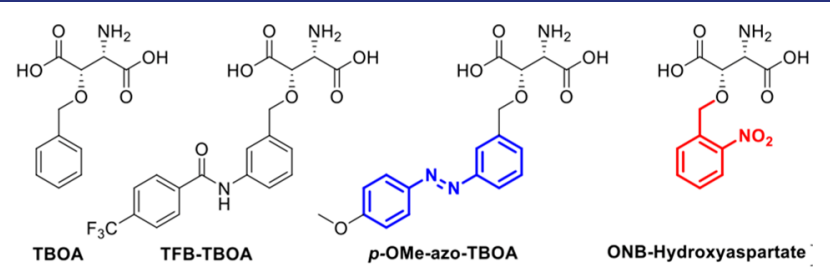

Figure 1. Structures of inhibitors and photoactive compounds. The azobenzene part of the photoswitch and the o-nitrobenzyl (ONB) part of the caged compound are highlighted in blue and red, respectively. blue) and diarylethene molecules, ${ }^{15}$ or photocages, ${ }^{16}$ such as $o$ nitrobenzyl (ONB, Figure 1, highlighted in red), coumarin, or BODIPY derivatives, ${ }^{17}$ into the structure of bioactive compounds. The azobenzene scaffold has two forms: a thermally stable, linear trans isomer and a metastable, bent cis isomer. These forms, which can be reversibly interconverted (switched) by using light, differ in shape and properties, which translates to differences in biological activity of compounds to which the switches have been introduced. Importantly, the cisform is thermally unstable, and in time reverts to the stable trans form, with the half-life ranging from milliseconds to years. This dynamic behavior poses a major challenge for any structural investigation of photoswitchable azobenzene-based systems.

Another photopharmacological approach uses temporary deactivation of a biologically active small molecule with a photocage (photoremovable protecting group, PPG), resulting

Received: October 29, 2020

Published: January 15, 2021 
in a so-called "caged" compound. ${ }^{18}$ Irradiation breaks the bond between the PPG and the compound, releasing the ligand. This irreversible process triggers the biological response with a high spatiotemporal resolution and can be used for time-resolved structural studies on the uncaging event and the following protein response.

One of the key challenges of photopharmacology lies in the very limited understanding of the structural basis of the alterations in biological effects induced by photoswitching, which hampers our ability to optimize the potency and difference in activity between the two isomers. Achieving large differences in activity between isomers is crucial for accomplishing spatial-temporal control over drug activity, and the future success of photopharmacology relies on elucidation of the structural aspects for the photocontrol of biological activity. However, up to date, only a few structures of soluble proteins in complex with noncovalently bound photoswitchable inhibitors are described, ${ }^{19-22}$ but none for membrane proteins, although the use of photoswitches is actively pursued in these proteins as seen in the recent study of TRPV6 channels. ${ }^{23}$ Furthermore, to the best of our knowledge, there is not a single report on the structures of both isomers of an azobenzene-based small molecule photopharmacological agent bound to its target.

Here we report on crystallographic analysis of the binding mode of two photopharmacological agents, the photoswitchable $p$-OMe-azo-TBOA and photocaged ONB-hydroxyaspartate (Figure 1), to the membrane transporter $\mathrm{Glt}_{\mathrm{Tk}}$.

This protein belongs to the SLC1A family of transporters, which also includes seven human transporters: the glutamate or excitatory amino acid transporters EAAT1-5 and the neutral amino acid exchangers ASCT 1 and ASCT2. ${ }^{24}$ EAATs remove the neurotransmitter glutamate from the synaptic cleft to prevent overstimulation of glutamate receptors and related neurotoxic effects, and malfunctioning of these transporters is linked to several diseases. ${ }^{25-29}$ Glutamate transport is strictly coupled to cotransport of three sodium ions and a proton and counter transport of a potassium ion. Hence, directionality is dictated by the electrochemical gradients of these cations across the membrane. Under ischemic conditions and epilepsy, when the gradients are disrupted, glutamate transporters can function in reverse filling up the synaptic cleft with an excess of glutamate, thus causing severe neurotoxicity. ${ }^{24}$ This reverse transport is mainly ascribed to EAAT3 protein; ${ }^{30-32}$ hence, the selective temporary blockage of these transporters could be helpful. Although in the majority of EAAT-linked diseases the most promising route is enhancing the transport function (e.g., via increased expression of EAATs), there are some indications that inhibition of glutamate transporters can be used in the treatment of chronic pain. ${ }^{33}$ Furthermore, some disorders are linked to gain-of-function mutations of the chloride channel activity, e.g., P290R mutation in EAAT1 causing episodic ataxia. ${ }^{34}$ Hence, these transporters are considered as potential drug targets. The archaeal homologues $\mathrm{Glt}_{\mathrm{Ph}}$ from Pyrococcus horikoshii and Glt $\mathrm{Tk}_{\mathrm{Tk}}$ from Thermococcus kodakarensis are widely used as model systems to study the transport mechanism of the glutamate transporter family. ${ }^{35-43}$

Glutamate transporters are homotrimeric membrane proteins, where each protomer has a trimerization scaffold domain that anchors the protein in the membrane and a transport domain that binds the substrate and cations and transports them across the membrane via an elevator mechanism. ${ }^{37,40,44}$ Over the years, numerous crystal and cryo-EM structures of archaeal homologues have been obtained, revealing several conformations that the transporters successively adopt during the transport cycle, including outward- and inward-facing states, ${ }^{35,37,40}$ as well as providing insight in the cationsubstrate coupling mechanism. ${ }^{38,39}$ The transport activity of glutamate transporters can be blocked by different molecules, ${ }^{45-53}$ including competitive nontransported inhibitors DLthreo- $\beta$-benzyloxyaspartic acid (TBOA, Figure 1) and (3S)-3[[3-[[4-(trifluoromethyl)benzoyl] amino] phenyl] methoxy $]-\mathrm{L}-$ aspartic acid (TFB-TBOA, Figure 1). These inhibitors were also used for structural studies that provided details on the transport mechanism. When bound to the substrate-binding site of the glutamate transporter homologues $\mathrm{Glt}_{\mathrm{Ph}}$ and $\mathrm{Glt}_{\mathrm{Tk}}$, TBOA prevents closure of the HP2 loop that serves as an extracellular gate, ${ }^{36,40}$ which was also shown in the crystal structure of EAAT 1 complexed with TFB-TBOA. ${ }^{54}$ A cryo-EM structure of a mutant of the neutral amino acid exchanger ASCT2 from Homo sapiens ( $50 \%$ sequence identity to EAAT1) in complex with TBOA revealed that the same HP2 loop also works as an intracellular gate. ${ }^{55}$

Azobenzene-based photoswitchable inhibitors of glutamate transporters showed successful inhibition of mammalian EAATs. ${ }^{56}$ As most of structural studies of glutamate transporters have been done on their archaeal homologues, photoswitchable inhibitors tested on these proteins provide an important tool for understanding the biological effect of photoswitching. Recently, we have reported on the development of photoswitchable inhibitor $p$-OMe-azo-TBOA (Figures 1 and 2) based on the potent inhibitor TFB-TBOA $\left(\mathrm{IC}_{50}=0.4\right.$

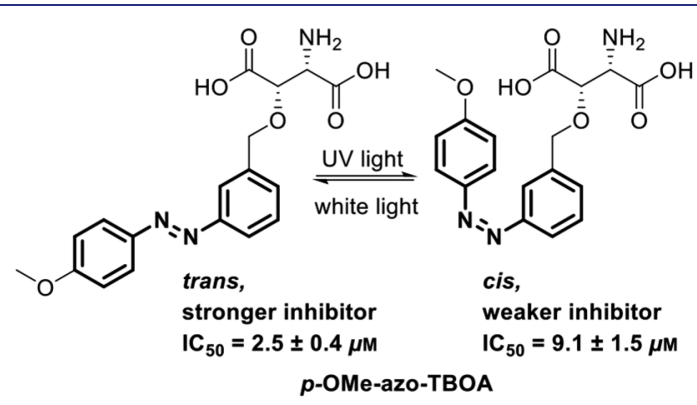

Figure 2. Photoswitch isomerization. Structures of $p$-OMe-azo-TBOA in trans (thermal) and cis (irradiated) configurations.

$\pm 0.1 \mu \mathrm{M}$ for transport by $\left.\mathrm{Glt}_{\mathrm{Tk}}\right) .{ }^{57} \mathrm{We}$ investigated the binding of trans and cis isomers of $p$-OMe-azo-TBOA to the Glt $_{\mathrm{Tk}}$ transporter. $p$-OMe-azo-TBOA in its thermally stable trans form readily binds to $\mathrm{Glt}_{\mathrm{Tk}}$ with an affinity in the low micromolar range. It can be reversibly switched in solution with UV light to its cis form, which results in a 3.6-fold decrease in inhibitory activity toward $\mathrm{Glt}_{\mathrm{Tk}} \cdot{ }^{57}$ In solution, this switching process is highly efficient and provides $>95 \%$ of the less potent cis isomer, which has a long half-life of $>10 \mathrm{~h}$. Switching the compounds back to the trans isomer with white light leads to the recovery of the higher potency (Figure 2). Altogether, this compound has desirable characteristics for structural studies because of the high trans-cis switching efficiency and long cis form half-life, despite a moderate difference in potency found in transport assays.

To establish the structural basis for this behavior, we report here the crystal structures of glutamate transporter homologue Glt $_{\mathrm{Tk}}$ in complex with $p$-OMe-azo-TBOA in both nonirradiated and irradiated states. Additionally, we obtained a 
structure of $\mathrm{Glt}_{\mathrm{Tk}}$ with a photocaged compound ONBhydroxyaspartate, which serves as an inhibitor in the nonirradiated state and generates $\beta$-hydroxyaspartate after uncaging, which is a substrate of mammalian glutamate transporters ${ }^{58}$ and its prokaryotic homologues. ${ }^{59,60}$

\section{RESULTS}

Glt $_{\text {Tk }}$ Crystal Structures in Complex with Photoswitches. The crystal structures of $\mathrm{Glt}_{\mathrm{Tk}}$ in complex with the trans or cis isomer of $p$-OMe-azo-TBOA (Table S1) each showed an outward-facing open state of the transporter. The inhibitor is bound in such a way that its aspartate moiety resides in the substrate binding site of the protein, while the photoswitchable part sticks out of the pocket (Figure 3). In

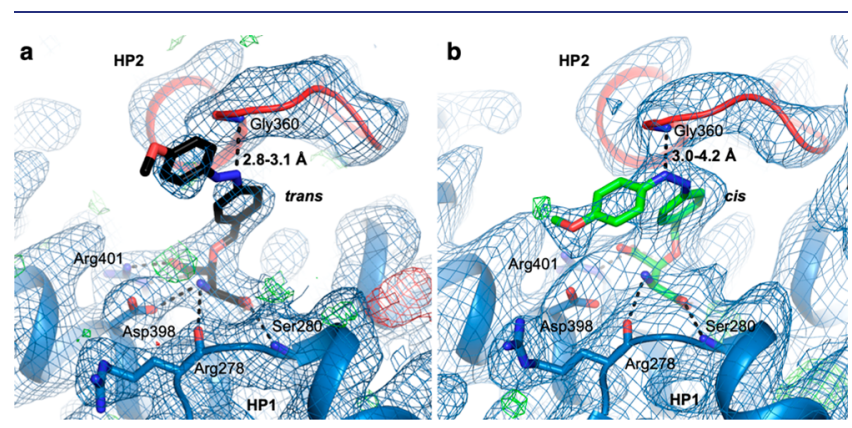

Figure 3. Substrate-binding site of $\mathrm{Glt}_{\mathrm{Tk}}$ with $p$-OMe-azo-TBOA in (a) trans and (b) cis configurations. $2 F_{\mathrm{o}}-F_{\mathrm{c}}$ electron density map contoured at $1 \sigma$ is shown as a blue mesh; $F_{\mathrm{o}}-F_{\mathrm{c}}$ map contoured at $\pm 3 \sigma$ is shown as a green-red mesh. The open HP2 loop is shown in red. Photoisomers are shown as black (trans) or green (cis) sticks. Possible hydrogen bonds (distances in $\AA$ ) between the binding site residues and the ligand are represented by black dashed lines.

this binding mode, the photoswitchable part occupies the space where the HP2 loop would have to move for gate closure, which in turn is required for subsequent conformational changes leading to transport. Therefore, $p$-OMe-azoTBOA inhibits transport by preventing closure of the extracellular gate, similar to what was observed in $\mathrm{Glt}_{\mathrm{Ph}}$ and $\mathrm{Glt}_{\mathrm{Tk}}$ with bound TBOA. ${ }^{36,40}$ While the orientation of the TBOA moiety of the $p$-OMe-azo-TBOA is very similar for both isomers, the photoswitchable parts show differences. The trans configuration of the photoswitch is mostly elongated, while the cis form has a bend in the diazo bond, which causes a shift of the $p$-OMe-benzyl group toward the HP1 loop (Figure 3).

Comparison of the $\mathrm{Glt}_{\mathrm{Tk}}-\mathrm{p}$-OMe-azo-TBOA complex structures with the crystal structure of the $\mathrm{Glt}_{\mathrm{Ph}}-\mathrm{TBOA}$ complex ${ }^{36}$ and $\mathrm{Glt}_{\mathrm{Tk}}-\mathrm{TBOA}$ complex ${ }^{40}$ shows that the presence of an additional azobenzene moiety in the ligand and its isomerization did not affect the HP2 conformation in its open state. The loop adopts an identical conformation in $\mathrm{Glt}_{\mathrm{Tk}}$ bound to either the cis or trans isomer and $\mathrm{Glt}_{\mathrm{Ph}}$ and $\mathrm{Glt}_{\mathrm{Tk}}$ bound to TBOA, with the rmsd values below $0.4 \AA$ (for $\mathrm{C} \alpha$ atoms, residues 355-361, Table S2). Therefore, more potent inhibition by the trans isomer in comparison with the cis isomer can be explained only by a higher affinity of the inhibitor in the trans state for the protein. Binding affinities of the compound to $\mathrm{Glt}_{\mathrm{Tk}}$, determined by isothermal titration calorimetry, are $1.89 \pm 1.26$ and $3.19 \pm 0.49 \mu \mathrm{M}$ for the trans and cis state, respectively. ${ }^{57}$ The crystal structure reveals that the main-chain amide group of Gly360 in HP2 forms a hydrogen bond with the azobenzene nitrogen of the ligand when it is in a trans configuration (distance 2.8-3.1 $\AA$ ). Isomerization of the diazo $(\mathrm{N}=\mathrm{N})$ bond from the trans to the cis configuration leads to a shift of the $p$-OMe-azo group away from the HP2 loop. The increased distance between the mainchain nitrogen atom of Gly360 and the azo-nitrogen atom of the compound (3.0-4.2 $\AA$ for the cis state, the range indicates that the compound is bound not uniformly to all three protomers) weakens or breaks this bond. Although in the cis configuration the $p$-OMe-azo group moves toward the HP1 loop, its oxygen is still too far away to form any additional polar contacts with the protein residues. Hence, the difference in affinity of the two photoisomers is likely explained by formation of an extra hydrogen bond for the trans-isomer in comparison with the cis-isomer.

Comparison of the inhibitor orientations in the different protomers of the homotrimeric protein using calculated omit maps (Figure S1) reveals that the photoswitchable moiety probably assumes a series of (slightly) different orientations in both the cis and trans configuration, reflecting its inherent flexibility. Alternatively, this might be a manifestation of an incomplete isomerization, similarly to what has been recently reported for reversibly switchable fluorescent proteins. ${ }^{61}$

To further investigate the structural basis for the lightinduced modulation of inhibitory potency, we additionally performed docking studies with the cocrystallized ligands and photoswitchable compounds that we studied earlier by transport assays. ${ }^{57}$ The selected structures showed better docking score for the trans than the cis conformation for five out of seven ligands (Figure S2). We attempted to find a correlation between the differences in docking scores for cis and trans conformations of ligands and the differences in their inhibition potency according to Hoorens et al. For the ligand with the largest significant inhibition difference obtained experimentally, $p$-MeO-azo-TBOA, also used here for crystallization, the docking score difference is also the largest, supporting the previously obtained experimental results. Docking of other ligands also revealed the preference for trans conformation, but such differences were not so obvious in the experimental transport assays ${ }^{57}$ (Figure S2). Interestingly the obtained poses of $p$-MeO-azo-TBOA by docking did not correspond well with the ligands' positions in the crystal structures (Figure S2), which might be either a result of shortcomings of our in silico calculations or the manifestation of site-specific radiation-induced damage of ligands. This was also observed by others, for example, in the crystal structure of ligand binding domain of a glutamate receptor GluK2 in complex with the photoswitchable compound Gluazo. ${ }^{22}$ Alternatively, the influence of buffer composition (in this case $40 \%$ PEG 400 was used for cryoprotection) could have affected the ligand binding pose, which has been reported for other ligands.

Interestingly, according to our docking calculations, the difference in binding affinities can be attributed to an additional hydrogen bond formation in the trans state as well, albeit between different atoms, namely the $p$-OMe group of the inhibitor and main-chain nitrogens of Leu154 and Val153 (Figure S2).

Glt $_{\text {Tk }}$ Crystal Structure in Complex with a Photocaged Inhibitor. The identical position of the HP2 loop in the presence of the cis and trans isomer of the inhibitor poses challenges to any future time-resolved measurements, as it could lead to ambiguity in the interpretation of the structural 
data. To overcome this issue, we also crystallized $\mathrm{Glt}_{\mathrm{Tk}}$ in complex with the photocaged compound ONB-hydroxyaspartate (Figures 1, 4, and 5), which does not suffer from potential
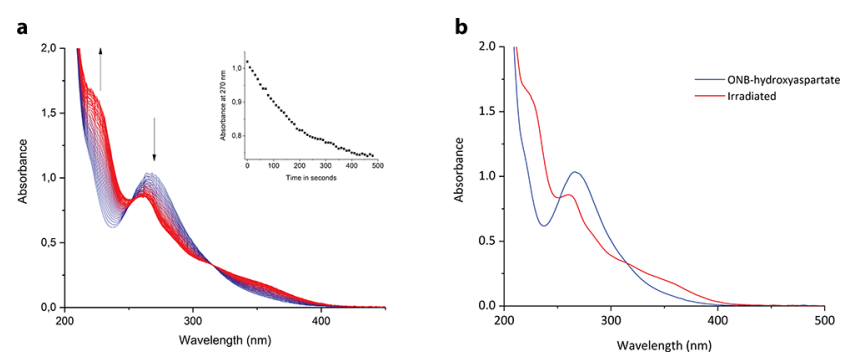

Figure 4. UV-vis absorption spectra of ONB-hydroxyaspartate in $\mathrm{H}_{2} \mathrm{O}$ $(237 \mu \mathrm{M})$ under UV-irradiation $\left(t_{\text {irr }}=300 \mathrm{~nm}\right.$, photon flux $=3.25 \times$ $\left.10^{-9} \mathrm{~mol} \mathrm{~s}^{-1}\right) \cdot t_{\text {irr }}=480 \mathrm{~s}, T=293 \mathrm{~K}$. Right: before and after irradiation.

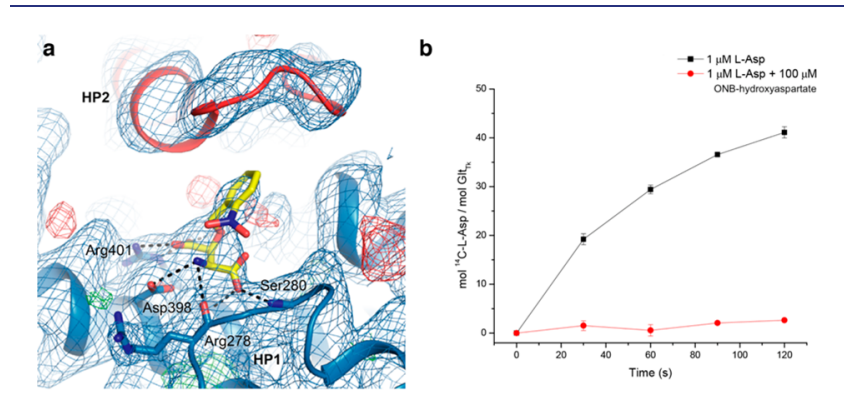

Figure 5. Caged compound (ONB-hydroxyaspartate) binding to $\mathrm{Glt}_{\mathrm{Tk}}$. (a) Interactions in the binding site (coloring is the same as in Figure 3). (b) Radioactive aspartate uptake in the presence and absence of the caged compound.

problems related to cis-trans isomerization. In the nonirradiated form, this compound is a close structural analogue of TFB-TBOA (Figure 1), and we expected it to act as an inhibitor. Exposure to UV light cleaves the PPG and releases the hydroxyaspartate, which is a known substrate for transport. $^{58-60}$

The ONB-hydroxyaspartate was prepared via a nine-step chemoenzymatic synthesis route by using an engineered variant of methylaspartate ammonia lyase (MAL-L384A) as a biocatalyst (for experimental details, see the Supporting Information). ${ }^{62,63}$ Its UV-vis spectrum features a band at $\lambda$ $=270 \mathrm{~nm}$ (Figure 4), characteristic for the ONB system. Irradiation of ONB-hydroxyaspartate at $\lambda=312 \mathrm{~nm}$ leads to the drop of absorbance of this band, indicative of the uncaging of the transport substrate. In water, this process (Figure 4) proceeds with a quantum yield of $28 \%$, which translates to a high uncaging cross section (a product of quantum yield and molar attenuation coefficient $\left.\varepsilon_{300}=2138 \mathrm{M}^{-1} \mathrm{~cm}^{-1}\right)$ of 599 $\mathrm{M}^{-1} \mathrm{~cm}^{-1}$.

The obtained crystal structure (Figure 5) is essentially identical with the $\mathrm{Glt}_{\mathrm{Tk}}$-p-OMe-azo-TBOA structures ( $\mathrm{rmsd}$ of $0.3 \AA$, all $\mathrm{C} \alpha$ atoms). Similar to the structures with photoswitchable compound isomers, the density for the TBOA-equivalent part of the photocaged ligand is clearly observed in the omit map (Figure S1). This is indicative that the photocaged compound is likely a competitive inhibitor. ONB-hydroxyaspartate indeed inhibited uptake of radiolabeled aspartate into proteoliposomes reconstituted with purified Glt $_{\text {Tk }}$ (Figure 5).

\section{DISCUSSION}

The activity of glutamate transporters can be reversibly controlled by using photoswitchable TBOA derivatives, ${ }^{57,64}$ with thermally adapted trans $p$-OMe-azo-TBOA inhibiting more potently than the cis isomer. ${ }^{57}$ To obtain structural insight into the mode of action of the photoswitchable TBOA derivative $p$-OMe-azo-TBOA and a new photocaged compound ONB-hydroxyaspartate, we performed cocrystallization experiments with $\mathrm{Glt}_{\mathrm{Tk}}$. TBOA-like inhibitors are nontransportable blockers of glutamate transporters that inhibit transport by preventing closure of the gate formed by the HP2 loop. ${ }^{36,54,55}$ We solved two crystal structures of $\mathrm{Glt}_{\mathrm{Tk}}$ in the complex with $p$-OMe-azo-TBOA, after thermal relaxation or irradiation of the crystals, producing the trans and cis isomers of the compound, respectively, and one structure of the protein bound to the photocaged ONB-hydroxyaspartate. In all three structures, Glt ${ }_{\mathrm{Tk}}$ was in an identical outward facing conformation with open HP2 gate (see Table S2 for rmsd values). The structures suggest that the conformation of the open gate reflects an intrinsically preferred state at least under our experimental conditions, regardless of the identity of the inhibitor present. Considering the absence of crystal contacts in the region of the gate, it is unlikely that its conformation is enforced by the crystal environment. In the crystal structure of Glt $_{\mathrm{Ph}}$ bound to $\mathrm{TBOA}^{36}$ as well as in the recent cryo-EM structure of $\mathrm{Glt}_{\mathrm{Tk}}$ with TBOA, ${ }^{40}$ a similar conformation of HP2 was found, which further indicates that such an open gate conformation represents a defined low-energy state.

Analysis of the two $\mathrm{Glt}_{\mathrm{Tk}}$ structures with bound $p$-OMe-azoTBOA provides a possible explanation for the different inhibitory effect of the trans and cis isomers. An extra hydrogen bond between the HP2 loop and the inhibitor in the trans configuration, compared to the cis isomer, could explain the higher binding affinity and stronger inhibitory properties of the trans-p-OMe-azo-TBOA. Because the isomerization of the ligand did not affect the extent of opening of the external HP2 gate, it is likely that the different extent of inhibitory potency of the cis- and trans-isomer is entirely due to the difference in binding affinity.

The structures of nonirradiated and irradiated $p$-OMe-azoTBOA bound to $\mathrm{Glt}_{\mathrm{Tk}}$ showed configurations of the photoswitch in its final isomeric states. However, the place where the photoswitch isomerizes remains unknown, and the process could go via two possible scenarios: (i) photoisomerization could have taken place while the compound was bound to the protein, or (ii) the trans-form could have dissociated and then isomerized in the solvent followed by association of the cisform back to the protein. Future time-resolved X-ray crystallography studies may allow the distinction between these scenarios by testing whether isomerization takes place while the inhibitor is bound to the protein in the crystal.

The identical position of the HP2 loop in the presence of the cis and trans isomer of the inhibitor poses challenges to any future time-resolved measurements of protein dynamics. Therefore, we also solved the structure of $\mathrm{Glt}_{\mathrm{Tk}}$ in complex with the photocaged compound ONB-hydroxyaspartate, which (i) readily binds to the transporter and (ii) can be irradiated to generate a transported substrate. Because gate closure is an essential conformational change for transport, uncaging of ONB-hydroxyaspartate can be a useful tool to study the dynamics of gate movement in SLC1A transporters with high 
time resolution since the caging allows precise and controlled photo-release of a substrate to trigger the reaction.

The design of photopharmacological agents is most often focused on the analysis of the bioactive compound and defining the molecular motifs that can be substituted with a photoswitch to enable control of activity with light. ${ }^{65}$ Recently, computational molecular modeling also enables protein-based approaches in which the structure of the target is taken into consideration, ${ }^{10,19,66,67}$ albeit mostly to explain the observed effects rather than to predict them. The structural information about differential binding of the two isomers of a photopharmacological agent, presented here, constitutes the next step in structural evaluation that shows for the first time how a photopharmacological agent binds in its two forms to the target and modulates its activity in a light-controlled manner. We believe that this insight will empower the future development of target-focused design principles for photopharmacology.

\section{MATERIALS AND METHODS}

Protein Purification and Crystallization. $\mathrm{His}_{8}$-tagged wild-type $\mathrm{Glt}_{\mathrm{Tk}}$ was purified in the apo state in $10 \mathrm{mM}$ Hepes $\mathrm{KOH}, \mathrm{pH} 8.0$, $100 \mathrm{mM} \mathrm{KCl}, 0.15 \% \mathrm{DM}$ as it is described in refs 39 and 40 . Concentrated to $\sim 7 \mathrm{mg} \mathrm{mL} \mathrm{mL}^{-1}$ (Vivaspin 500, $50000 \mathrm{MWCO}$, Vivaproducts), the protein sample was supplemented with $300 \mathrm{mM}$ $\mathrm{NaCl}$.

Dissolved in water, $p$-OMe-azo-TBOA was preheated at $60{ }^{\circ} \mathrm{C}$ for $10 \mathrm{~min}$ to generate the thermal (trans) state and added to a protein solution to $360 \mu \mathrm{M}$ concentration (with final protein concentration $120 \mu \mathrm{M})$. Crystals of the protein with $p$-OMe-azo-TBOA were obtained in the dark in 23\% PEG 400, $100 \mathrm{mM}$ sodium acetate, $\mathrm{pH}$ $4.8,50 \mathrm{mM} \mathrm{NaCl}, 20 \mathrm{mM} \mathrm{MgCl} 2$ at $4{ }^{\circ} \mathrm{C}$ by using the hanging drop vapor diffusion technique. Crystallization conditions supplemented with $10 \%$ glycerol were used as a cryoprotectant. For the cis-state, crystals with the $p$-OMe-azo-TBOA in the trans state were irradiated with $365 \mathrm{~nm}$ UV-lamp for $1 \mathrm{~min}^{57}$ and treated with cryoprotectant in red light to avoid further isomerization.

Crystals of $\mathrm{Glt}_{\mathrm{Tk}}$ with the caged compound were obtained in the dark in 20\% PEG 400, $250 \mathrm{mM}$ glycine, $\mathrm{pH} 9.2,50 \mathrm{mM} \mathrm{MgCl}_{2}$ at 4 ${ }^{\circ} \mathrm{C}$. The protein and ligand concentrations were 115 and $430 \mu \mathrm{M}$, respectively. For cryoprotection, crystals were soaked in crystallization conditions containing 40\% PEG 400 under the red light.

Ligand Docking. For each model, a single chain for docking studies was selected based on $2 F_{\mathrm{o}}-F_{\mathrm{c}}$ maps quality around ligand and surrounding residues (in both cases chain A). Four models (chain A with and without sodium ions for both trans and cis structures) were generated to assess whether they can reflect $\mathrm{Glt}_{\mathrm{Tk}}$ inhibition data. ${ }^{57}$ The molecular docking was performed by using ICM Molsoft Pro (ver. 3.9-1b). The cocrystallized ligands were removed, and structures were converted into ICM format with default settings, which includes building of any missing side chains, addition of hydrogens, and energy-based Gln/Asn/His conformation optimization. A docking box for all four models was selected around the crystallographic ligand position in the cis conformation. We repeated docking studies five times with effort (ligand sampling depth), ${ }^{35}$ each time saving three best conformations for each ligand. Docking scores were exported by using rdkit (ver. 2020.03.6) and analyzed by using pandas (ver. 1.1.2) and seaborn (0.11.0). Among all models, cis_a_Na (crystal structure with ligand bound in cis conformation, A chain, $\mathrm{Na}^{+}$ions present) showed a better docking score for the trans conformation of the ligand for five out of seven ligands (less for others), and poses generated by docking in this model were analyzed visually.

Data Collection and Structure Determination. Crystals were flash-frozen in liquid nitrogen, and data were collected at the PETRA III beamlines P13 and P14 (EMBL/DESY, Hamburg). Data were processed with XDS, ${ }^{68}$ and the structures were solved by Molecular
Replacement with Phaser ${ }^{69}$ by using Glt $_{\mathrm{Tk}}$ model PDB ID 5E9S. Manual modification of the structure was performed in $\mathrm{COOT}^{70}$ and refinement in Phenix refine. ${ }^{71}$ Refined models were deposited into PDB under accession codes 6ZLH, 6ZL4, and 6ZGB for Glt $\mathrm{Tk}_{\mathrm{T}}$ with $p$ OMe-azo-TBOA trans- and cis-state and the caged compound, respectively. The final refinement statistics of the models are summarized in Table S1.

Transport Assay. Reconstitution of purified $\mathrm{Glt}_{\mathrm{Tk}}$ into proteoliposomes and activity assays were performed as described previously. ${ }^{57}$

\section{ASSOCIATED CONTENT}

Supporting Information

The Supporting Information is available free of charge at https://pubs.acs.org/doi/10.1021/jacs.0c11336.

Tables S1 and S2; Figures S1-S4, and information about synthesis (PDF)

\section{Accession Codes}

The structural models and associated structure factors are available at PDB data bank under the following accession codes: 6ZLH, 6ZL4, and 6ZGB.

\section{AUTHOR INFORMATION}

\section{Corresponding Authors}

Wiktor Szymanski - University Medical Center Groningen, Department of Radiology, Medical Imaging Center and Stratingh Institute for Chemistry, Faculty of Science and Engineering, University of Groningen, 9713 GZ Groningen, The Netherlands; Email: w.c.szymanski@rug.nl

Dirk J. Slotboom - Groningen Biomolecular Sciences and Biotechnology Institute, University of Groningen, 9747 AG Groningen, The Netherlands; Email: d.j.slotboom@rug.nl

Albert Guskov - Groningen Biomolecular Sciences and Biotechnology Institute, University of Groningen, 9747 AG Groningen, The Netherlands; Research Center for Molecular Mechanisms of Aging and Age-related Diseases, Moscow Institute of Physics and Technology, Dolgoprudny 141701, Russia; O orcid.org/0000-0003-2340-2216; Email: a.guskov@rug.nl

\section{Authors}

Valentina Arkhipova - University Medical Center Groningen, Department of Radiology, Medical Imaging Center, University of Groningen, 9713 GZ Groningen, The Netherlands; Groningen Biomolecular Sciences and Biotechnology Institute, University of Groningen, 9747 AG Groningen, The Netherlands

Haigen Fu - Department of Chemical and Pharmaceutical Biology, Groningen Research Institute of Pharmacy, University of Groningen, 9713 AV Groningen, The Netherlands

Mark W. H. Hoorens - University Medical Center Groningen, Department of Radiology, Medical Imaging Center and Stratingh Institute for Chemistry, Faculty of Science and Engineering, University of Groningen, 9713 GZ Groningen, The Netherlands

Gianluca Trinco - Groningen Biomolecular Sciences and Biotechnology Institute, University of Groningen, 9747 AG Groningen, The Netherlands

Lucien N. Lameijer - University Medical Center Groningen, Department of Radiology, Medical Imaging Center and Stratingh Institute for Chemistry, Faculty of Science and 
Engineering, University of Groningen, 9713 GZ Groningen, The Netherlands

Egor Marin - Groningen Biomolecular Sciences and Biotechnology Institute, University of Groningen, 9747 AG Groningen, The Netherlands; Research Center for Molecular Mechanisms of Aging and Age-related Diseases, Moscow Institute of Physics and Technology, Dolgoprudny 141701, Russia

Ben L. Feringa - Stratingh Institute for Chemistry, Faculty of Science and Engineering, University of Groningen, 9747 AG Groningen, The Netherlands; 10 orcid.org/0000-0003-05888435

Gerrit J. Poelarends - Department of Chemical and Pharmaceutical Biology, Groningen Research Institute of Pharmacy, University of Groningen, 9713 AV Groningen, The Netherlands; (1) orcid.org/0000-0002-6917-6368

Complete contact information is available at:

https://pubs.acs.org/10.1021/jacs.0c11336

\section{Funding}

The work was supported by NWO ECHO Grant 711.017.012 to W.S. and D.J.S. and Grant NWO OCENW.KLEIN.141 to A.G.

\section{Notes}

The authors declare no competing financial interest.

\section{ACKNOWLEDGMENTS}

H.F. acknowledges funding from the China Scholarship Council; E.M. and A.G. acknowledge support from RFBR (19-29-12022).

\section{ABBREVIATIONS}

$\mathrm{Glt}_{\mathrm{Tk}}$ glutamate transporter homologue from Thermococcus kodakarensis; $\mathrm{Glt}_{\mathrm{Ph}}$, glutamate transporter homologue from Phyrococcus horikoshii; SLC, solute carrier; GPCR, G-protein coupled receptor; ONB, o-nitrobenzyl; BODIPY, boron dipyrromethene; PPG, photoremovable protecting group; TRPV, transient receptor potential vanilloid channel; EAAT, excitatory amino acid transporter; ASCT, alanine-serinecystein transporter; TBOA, DL-threo- $\beta$-benzyloxyaspartic acid; TFB-TBOA, (3S)-3-[[3-[[4-(trifluoromethyl)benzoyl] amino]phenyl]methoxy]-L-aspartic acid.

\section{REFERENCES}

(1) Velema, W. A.; Van Der Berg, J. P.; Hansen, M. J.; Szymanski, W.; Driessen, A. J. M.; Feringa, B. L. Optical Control of Antibacterial Activity. Nat. Chem. 2013, 5 (11), 924-928.

(2) Borowiak, M.; Nahaboo, W.; Reynders, M.; Nekolla, K.; Jalinot, P.; Hasserodt, J.; Rehberg, M.; Delattre, M.; Zahler, S.; Vollmar, A.; Trauner, D.; Thorn-Seshold, O. Photoswitchable Inhibitors of Microtubule Dynamics Optically Control Mitosis and Cell Death. Cell 2015, 162 (2), 403-411.

(3) Hoorens, M. W. H.; Szymanski, W. Reversible, Spatial and Temporal Control over Protein Activity Using Light. Trends Biochem. Sci. 2018, 43 (8), 567-575.

(4) Lerch, M. M.; Hansen, M. J.; van Dam, G. M.; Szymanski, W.; Feringa, B. L. Emerging Targets in Photopharmacology. Angew. Chem., Int. Ed. 2016, 55 (37), 10978-10999.

(5) Tochitsky, I.; Kienzler, M. A.; Isacoff, E.; Kramer, R. H. Restoring Vision to the Blind with Chemical Photoswitches. Chem. Rev. 2018, 118 (21), 10748-10773.

(6) Hüll, K.; Morstein, J.; Trauner, D. In Vivo Photopharmacology. Chem. Rev. 2018, 118 (21), 10710-10747.
(7) Lundstrom, K. Structural Genomics and Drug Discovery: Molecular Pharmacology. J. Cell. Mol. Med. 2007, 11 (2), 224-238.

(8) Gomez-Santacana, X.; de Munnik, S. M; Mocking, T. A M; Hauwert, N. J; Sun, S.; Vijayachandran, P.; de Esch, I. J P; Vischer, H. F; Wijtmans, M.; Leurs, R. A Toolbox of Molecular Photoswitches to Modulate the CXCR3 Chemokine Receptor with Light. Beilstein J. Org. Chem. 2019, 15 (1), 2509-2523.

(9) Ricart-Ortega, M.; Font, J.; Llebaria, A. GPCR Photopharmacology. Mol. Cell. Endocrinol. 2019, 488, 36-51.

(10) Hauwert, N. J.; Mocking, T. A. M.; Da Costa Pereira, D.; Kooistra, A. J.; Wijnen, L. M.; Vreeker, G. C. M.; Verweij, E. W. E.; De Boer, A. H.; Smit, M. J.; De Graaf, C.; Vischer, H. F.; de Esch, I. J. P.; Wijtmans, M.; Leurs, R. Synthesis and Characterization of a Bidirectional Photoswitchable Antagonist Toolbox for Real-Time GPCR Photopharmacology. J. Am. Chem. Soc. 2018, 140 (12), 42324243.

(11) Bregestovski, P. D.; Maleeva, G. V. Photopharmacology: A Brief Review Using the Control of Potassium Channels as an Example. Neurosci. Behav. Physiol. 2019, 49 (2), 184-191.

(12) Sansalone, L.; Zhao, J.; Richers, M. T.; Ellis-Davies, G. C. R. Chemical Tuning of Photoswitchable Azobenzenes: A Photopharmacological Case Study Using Nicotinic Transmission. Beilstein J. Org. Chem. 2019, 15 (1), 2812-2821.

(13) Podewin, T.; Broichhagen, J.; Frost, C.; Groneberg, D.; Ast, J.; Meyer-Berg, H.; Fine, N. H. F.; Friebe, A.; Zacharias, M.; Hodson, D. J.; Trauner, D.; Hoffmann-Röder, A. Optical Control of a ReceptorLinked Guanylyl Cyclase Using a Photoswitchable Peptidic Hormone. Chem. Sci. 2017, 8 (6), 4644-4653.

(14) Beharry, A. A.; Woolley, G. A. Azobenzene Photoswitches for Biomolecules. Chem. Soc. Rev. 2011, 40 (8), 4422-4437.

(15) Babii, O.; Afonin, S.; Berditsch, M.; Rei $\beta$ er, S.; Mykhailiuk, P. K.; Kubyshkin, V. S.; Steinbrecher, T.; Ulrich, A. S.; Komarov, I. V. Controlling Biological Activity with Light: Diarylethene-Containing Cyclic Peptidomimetics. Angew. Chem., Int. Ed. 2014, 53 (13), 33923395.

(16) Klán, P.; Šolomek, T.; Bochet, C. G.; Blanc, A.; Givens, R.; Rubina, M.; Popik, V.; Kostikov, A.; Wirz, J. Photoremovable Protecting Groups in Chemistry and Biology: Reaction Mechanisms and Efficacy. Chem. Rev. 2013, 113 (1), 119-191.

(17) Slanina, T.; Shrestha, P.; Palao, E.; Kand, D.; Peterson, J. A.; Dutton, A. S.; Rubinstein, N.; Weinstain, R.; Winter, A. H.; Klán, P. In Search of the Perfect Photocage: Structure-Reactivity Relationships in Meso-Methyl BODIPY Photoremovable Protecting Groups. J. Am. Chem. Soc. 2017, 139 (42), 15168-15175.

(18) Deiters, A. Principles and Applications of the Photochemical Control of Cellular Processes. ChemBioChem 2010, 11 (1), 47-53.

(19) Schehr, M.; Ianes, C.; Weisner, J.; Heintze, L.; Müller, M. P.; Pichlo, C.; Charl, J.; Brunstein, E.; Ewert, J.; Lehr, M.; Baumann, U.; Rauh, D.; Knippschild, U.; Peifer, C.; Herges, R. 2-Azo-, 2-DiazocineThiazols and 2-Azo-Imidazoles as Photoswitchable Kinase Inhibitors: Limitations and Pitfalls of the Photoswitchable Inhibitor Approach. Photochem. Photobiol. Sci. 2019, 18 (6), 1398-1407.

(20) Morgan, H. P.; McNae, I. W.; Nowicki, M. W.; Zhong, W.; Michels, P. A. M.; Auld, D. S.; Fothergill-Gilmore, L. A.; Walkinshaw, M. D. The Trypanocidal Drug Suramin and Other Trypan Blue Mimetics Are Inhibitors of Pyruvate Kinases and Bind to the Adenosine Site. J. Biol. Chem. 2011, 286 (36), 31232-31240.

(21) Dubay, K. H.; Iwan, K.; Osorio-Planes, L.; Geissler, P. L.; Groll, M.; Trauner, D.; Broichhagen, J. A Predictive Approach for the Optical Control of Carbonic Anhydrase II Activity. ACS Chem. Biol. 2018, 13 (3), 793-800.

(22) Reiter, A.; Skerra, A.; Trauner, D.; Schiefner, A. A Photoswitchable Neurotransmitter Analogue Bound to Its Receptor. Biochemistry 2013, 52 (50), 8972-8974.

(23) Cunha, M. R.; Bhardwaj, R.; Lindinger, S.; Butorac, C.; Romanin, C.; Hediger, M. A.; Reymond, J. L. Photoswitchable Inhibitor of the Calcium Channel TRPV6. ACS Med. Chem. Lett. 2019, 10 (9), 1341-1345. 
(24) Vandenberg, R. J.; Ryan, R. M. Mechanisms of Glutamate Transport. Physiol. Rev. 2013, 93 (4), 1621-1657.

(25) Stergachis, A. B.; Pujol-Giménez, J.; Gyimesi, G.; Fuster, D.; Albano, G.; Troxler, M.; Picker, J.; Rosenberg, P. A.; Bergin, A.; Peters, J.; El Achkar, C. M.; Harini, C.; Manzi, S.; Rotenberg, A.; Hediger, M. A.; Rodan, L. H. Recurrent SLC1A2 Variants Cause Epilepsy via a Dominant Negative Mechanism. Ann. Neurol. 2019, 85 (6), 921-926.

(26) Zike, I. D.; Chohan, M. O.; Kopelman, J. M.; Krasnow, E. N.; Flicker, D.; Nautiyal, K. M.; Bubser, M.; Kellendonk, C.; Jones, C. K.; Stanwood, G.; Tanaka, K. F.; Moore, H.; Ahmari, S. E.; VeenstraVanderWeele, J. OCD Candidate Gene SLC1A1/EAAT3 Impacts Basal Ganglia-Mediated Activity and Stereotypic Behavior. Proc. Natl. Acad. Sci. U. S. A. 2017, 114 (22), 5719-5724.

(27) Choi, K. D.; Jen, J. C.; Choi, S. Y.; Shin, J. H.; Kim, H. S.; Kim, H. J.; Kim, J. S.; Choi, J. H. Late-Onset Episodic Ataxia Associated with SLC1A3Mutation. J. Hum. Genet. 2017, 62 (3), 443-446.

(28) Heimer, G.; Marek-Yagel, D.; Eyal, E.; Barel, O.; Oz Levi, D.; Hoffmann, C.; Ruzzo, E. K.; Ganelin-Cohen, E.; Lancet, D.; Pras, E.; Rechavi, G.; Nissenkorn, A.; Anikster, Y.; Goldstein, D. B.; Ben Zeev, B. SLC1A4Mutations Cause a Novel Disorder of Intellectual Disability, Progressive Microcephaly, Spasticity and Thin Corpus Callosum. Clin. Genet. 2015, 88 (4), 327-335.

(29) Van Geldermalsen, M.; Wang, Q.; Nagarajah, R.; Marshall, A. D.; Thoeng, A.; Gao, D.; Ritchie, W.; Feng, Y.; Bailey, C. G.; Deng, N.; Harvey, K.; Beith, J. M.; Selinger, C. I.; O’Toole, S. A.; Rasko, J. E. J.; Holst, J. ASCT2/SLC1A5 Controls Glutamine Uptake and Tumour Growth in Triple-Negative Basal-like Breast Cancer. Oncogene 2016, 35 (24), 3201-3208.

(30) Gebhardt, C.; Körner, R.; Heinemann, U. Delayed Anoxic Depolarizations in Hippocampal Neurons of Mice Lacking the Excitatory Amino Acid Carrier 1. J. Cereb. Blood Flow Metab. 2002, 22 (5), 569-575.

(31) Bjørn-Yoshimoto, W.; Underhill, S. M. The Importance of the Excitatory Amino Acid Transporter 3 (EAAT3). Neurochem. Int. 2016, 98, 4-18.

(32) Rossi, D. J.; Oshima, T.; Attwell, D. Glutamate Release in Severe Brain Ischaemia Is Mainly by Reversed Uptake. Nature 2000, 403 (6767), 316-321.

(33) Yaster, M.; Guan, X.; Petralia, R. S.; Rothstein, J. D.; Lu, W.; Tao, Y. X. Effect of Inhibition of Spinal Cord Glutamate Transporters on Inflammatory Pain Induced by Formalin and Complete Freund's Adjuvant. Anesthesiology 2011, 114 (2), 412-423.

(34) Winter, N.; Kovermann, P.; Fahlke, C. A Point Mutation Associated with Episodic Ataxia 6 Increases Glutamate Transporter Anion Currents. Brain 2012, 135 (11), 3416-3425.

(35) Yernool, D.; Boudker, O.; Jin, Y.; Gouaux, E. Structure of a Glutamate Transporter Homologue from Pyrococcus Horikoshii. Nature 2004, 431 (7010), 811-818.

(36) Boudker, O.; Ryan, R. M.; Yernool, D.; Shimamoto, K.; Gouaux, E. Coupling Substrate and Ion Binding to Extracellular Gate of a Sodium-Dependent Aspartate Transporter. Nature 2007, 445 (7126), 387-393.

(37) Reyes, N.; Ginter, C.; Boudker, O. Transport Mechanism of a Bacterial Homologue of Glutamate Transporters. Nature 2009, 462 (7275), 880-885.

(38) Jensen, S.; Guskov, A.; Rempel, S.; Hänelt, I.; Slotboom, D. J. Crystal Structure of a Substrate-Free Aspartate Transporter. Nat. Struct. Mol. Biol. 2013, 20 (10), 1224-1226.

(39) Guskov, A.; Jensen, S.; Faustino, I.; Marrink, S. J.; Slotboom, D. J. Coupled Binding Mechanism of Three Sodium Ions and Aspartate in the Glutamate Transporter Homologue GltTk. Nat. Commun. 2016, 7, 13420.

(40) Arkhipova, V.; Guskov, A.; Slotboom, D. J. Structural Ensemble of a Glutamate Transporter Homologue in Lipid Nanodisc Environment. Nat. Commun. 2020, 11, 998.

(41) Alleva, C.; Kovalev, K.; Astashkin, R.; Berndt, M. I.; Baeken, C.; Balandin, T.; Gordeliy, V.; Fahlke, C.; Machtens, J. P. Na ${ }^{+}$-Dependent Gate Dynamics and Electrostatic Attraction Ensure Substrate
Coupling in Glutamate Transporters. Sci. Adv. 2020, 6 (47), eaba9854.

(42) Hall, J. L.; Sohail, A.; Cabrita, E. J.; Macdonald, C.; Stockner, T.; Sitte, H. H.; Angulo, J.; MacMillan, F. Saturation Transfer Difference NMR on the Integral Trimeric Membrane Transport Protein GltPh Determines Cooperative Substrate Binding. Sci. Rep. 2020, 10 (1), 1-9.

(43) Ruan, Y.; Miyagi, A.; Wang, X.; Chami, M.; Boudker, O.; Scheuring, S. Direct Visualization of Glutamate Transporter Elevator Mechanism by High-Speed AFM. Proc. Natl. Acad. Sci. U. S. A. 2017, $114,1584-1588$.

(44) Garaeva, A. A.; Slotboom, D. J. Elevator-Type Mechanisms of Membrane Transport. Biochem. Soc. Trans. 2020, 48 (3), 1227-1241.

(45) Esslinger, C. S.; Agarwal, S.; Gerdes, J.; Wilson, P. A.; Davis, E. S.; Awes, A. N.; O’Brien, E.; Mavencamp, T.; Koch, H. P.; Poulsen, D. J.; Rhoderick, J. F.; Chamberlin, A. R.; Kavanaugh, M. P.; Bridges, R. J. The Substituted Aspartate Analogue L- $\beta$-Threo-Benzyl-Aspartate Preferentially Inhibits the Neuronal Excitatory Amino Acid Transporter EAAT3. Neuropharmacology 2005, 49 (6), 850-861.

(46) Greenfield, A.; Grosanu, C.; Dunlop, J.; McIlvain, B.; Carrick, T.; Jow, B.; Lu, Q.; Kowal, D.; Williams, J.; Butera, J. Synthesis and Biological Activities of Aryl-Ether-, Biaryl-, and Fluorene-Aspartic Acid and Diaminopropionic Acid Analogs as Potent Inhibitors of the High-Affinity Glutamate Transporter EAAT2. Bioorg. Med. Chem. Lett. 2005, 15, 4985-4988.

(47) Jensen, A. A.; Erichsen, M. N.; Nielsen, C. W.; Stensbøl, T. B.; Kehler, J.; Bunch, L. Discovery of the First Selective Inhibitor of Excitatory Amino Acid Transporter Subtype 1. J. Med. Chem. 2009, 52 (4), 912-915.

(48) Hansen, S. W.; Erichsen, M. N.; Fu, B.; Bjørn-Yoshimoto, W. E.; Abrahamsen, B.; Hansen, J. C.; Jensen, A. A.; Bunch, L. Identification of a New Class of Selective Excitatory Amino Acid Transporter Subtype 1 (EAAT1) Inhibitors Followed by a StructureActivity Relationship Study. J. Med. Chem. 2016, 59 (19), 8757-8770.

(49) Abrahamsen, B.; Schneider, N.; Erichsen, M. N.; Huynh, T. H. V.; Fahlke, C.; Bunch, L.; Jensen, A. A. Allosteric Modulation of an Excitatory Amino Acid Transporter: The Subtype-Selective Inhibitor UCPH-101 Exerts Sustained Inhibition of EAAT1 through an Intramonomeric Site in the Trimerization Domain. J. Neurosci. 2013, 33 (3), 1068-1087.

(50) Balcar, V. J.; Johnston, G. A. R. The Structural Specificity of the High Affinity Uptake of L-Glutamate and L-Aspartate by Rat Brain Slices. J. Neurochem. 1972, 19 (11), 2657-2666.

(51) Shimamoto, K.; LeBrun, B.; Yasuda-Kamatani, Y.; Sakaitani, M.; Shigeri, Y.; Yumoto, N.; Nakajima, T. DL-Threo-BetaBenzyloxyaspartate, a Potent Blocker of Excitatory Amino Acid Transporters. Mol. Pharmacol. 1998, 53, 195-201.

(52) Shimamoto, K.; Sakai, R.; Takaoka, K.; Yumoto, N.; Nakajima, T.; Amara, S. G.; Shigeri, Y. Characterization of Novel L-Threo-BetaBenzyloxyaspartate Derivatives, Potent Blockers of the Glutamate Transporters. Mol. Pharmacol. 2004, 65 (4), 1008-1015.

(53) Fu, H.; Zhang, J.; Tepper, P. G.; Bunch, L.; Jensen, A. A.; Poelarends, G. J. Chemoenzymatic Synthesis and Pharmacological Characterization of Functionalized Aspartate Analogues as Novel Excitatory Amino Acid Transporter Inhibitors. J. Med. Chem. 2018, 61 (17), 7741-7753.

(54) Canul-Tec, J. C.; Assal, R.; Cirri, E.; Legrand, P.; Brier, S.; Chamot-Rooke, J.; Reyes, N. Structure and Allosteric Inhibition of Excitatory Amino Acid Transporter 1. Nature 2017, 544, 446-451.

(55) Garaeva, A. A.; Guskov, A.; Slotboom, D. J.; Paulino, C. A OneGate Elevator Mechanism for the Human Neutral Amino Acid Transporter ASCT2. Nat. Commun. 2019, 10 (1), 3427.

(56) Cheng, B.; Trauner, D.; Shchepakin, D.; Kavanaugh, M. P. A Photoswitchable Inhibitor of a Glutamate Transporter. ACS Chem. Neurosci. 2017, 8, 1668-1672.

(57) Hoorens, M. W. H.; Fu, H.; Duurkens, R. H.; Trinco, G.; Arkhipova, V.; Feringa, B. L.; Poelarends, G. J.; Slotboom, D. J.; Szymanski, W. Glutamate Transporter Inhibitors with PhotoControlled Activity. Adv. Ther. 2018, 1, 1800028. 
(58) Foster, A. C.; Li, Y.-X.; Runyan, S.; Dinh, T.; Venadas, S.; Chen, J.; Pashikanti, S.; Datta, A.; Ehring, G.; Staubli, U. Activity of the Enantiomers of Erythro-3-Hydroxyaspartate at Glutamate Transporters and NMDA Receptors. J. Neurochem. 2016, 136, 692-697.

(59) Tolner, B.; Ubbink-Kok, T.; Poolman, B.; Konings, W. N. Characterization of the Proton/Glutamate Symport Protein of Bacillus Subtilis and Its Functional Expression in Escherichia Coli. J. Bacteriol. 1995, 177 (10), 2863-2869.

(60) Tolner, B.; Ubbink-Kok, T.; Poolmann, B.; Konings, W. N. Cation-Selectivity of the L-Glutamate Transporters of Escherichia Coli, Bacillus Stearothermophilus and Bacillus Caldotenax: Dependence on the Environment in Which the Proteins Are Expressed. Mol. Microbiol. 1995, 18 (1), 123-133.

(61) Woodhouse, J.; Nass Kovacs, G.; Coquelle, N.; Uriarte, L. M.; Adam, V.; Barends, T. R. M.; Byrdin, M.; de la Mora, E.; Bruce Doak, R.; Feliks, M.; Field, M.; Fieschi, F.; Guillon, V.; Jakobs, S.; Joti, Y.; Macheboeuf, P.; Motomura, K.; Nass, K.; Owada, S.; Roome, C. M.; Ruckebusch, C.; Schirò, G.; Shoeman, R. L.; Thepaut, M.; Togashi, T.; Tono, K.; Yabashi, M.; Cammarata, M.; Foucar, L.; Bourgeois, D.; Sliwa, M.; Colletier, J.-P.; Schlichting, I.; Weik, M. Photoswitching Mechanism of a Fluorescent Protein Revealed by Time-Resolved Crystallography and Transient Absorption Spectroscopy. Nat. Commun. 2020, 11, 741.

(62) Fu, H.; Younes, S. H. H.; Saifuddin, M.; Tepper, P. G.; Zhang, J.; Keller, E.; Heeres, A.; Szymanski, W.; Poelarends, G. J. Rapid Chemoenzymatic Route to Glutamate Transporter Inhibitor L-TFBTBOA and Related Amino Acids. Org. Biomol. Chem. 2017, 15 (11), 2341-2344.

(63) Raj, H.; Szymański, W.; De Villiers, J.; Rozeboom, H. J.; Veetil, V. P.; Reis, C. R.; De Villiers, M.; Dekker, F. J.; De Wildeman, S.; Quax, W. J.; Thunnissen, A. M. W. H.; Feringa, B. L.; Janssen, D. B.; Poelarends, G. J. Engineering Methylaspartate Ammonia Lyase for the Asymmetric Synthesis of Unnatural Amino Acids. Nat. Chem. 2012, 4 (6), 478-484.

(64) Cheng, B.; Shchepakin, D.; Kavanaugh, M. P.; Trauner, D. Photoswitchable Inhibitor of a Glutamate Transporter. ACS Chem. Neurosci. 2017, 8 (8), 1668-1672.

(65) Morstein, J.; Awale, M.; Reymond, J. L.; Trauner, D. Mapping the Azolog Space Enables the Optical Control of New Biological Targets. ACS Cent. Sci. 2019, 5 (4), 607-618.

(66) Hoorens, M. W. H.; Ourailidou, M. E.; Rodat, T.; van der Wouden, P. E.; Kobauri, P.; Kriegs, M.; Peifer, C.; Feringa, B. L.; Dekker, F. J.; Szymanski, W. Light-Controlled Inhibition of BRAFV600E Kinase. Eur. J. Med. Chem. 2019, 179, 133-146.

(67) Xu, Z.; Shi, L.; Jiang, D.; Cheng, J.; Shao, X.; Li, Z. Azobenzene Modified Imidacloprid Derivatives as Photoswitchable Insecticides: Steering Molecular Activity in a Controllable Manner. Sci. Rep. 2015, $5,1-8$.

(68) Kabsch, W. Integration, Scaling, Space-Group Assignment and Post-Refinement. Acta Crystallogr., Sect. D: Biol. Crystallogr. 2010, 66 (2), 133-144.

(69) McCoy, A. J.; Grosse-Kunstleve, R. W.; Adams, P. D.; Winn, M. D.; Storoni, L. C.; Read, R. J. Phaser Crystallographic Software. J. Appl. Crystallogr. 2007, 40 (4), 658-674.

(70) Emsley, P.; Lohkamp, B.; Scott, W. G.; Cowtan, K. Features and Development of Coot. Acta Crystallogr., Sect. D: Biol. Crystallogr. 2010, 66 (4), 486-501.

(71) Afonine, P. V.; Grosse-Kunstleve, R. W.; Echols, N.; Headd, J. J.; Moriarty, N. W.; Mustyakimov, M.; Terwilliger, T. C.; Urzhumtsev, A.; Zwart, P. H.; Adams, P. D. Towards Automated Crystallographic Structure Refinement with Phenix.Refine. Acta Crystallogr., Sect. D: Biol. Crystallogr. 2012, 68 (4), 352-367. 